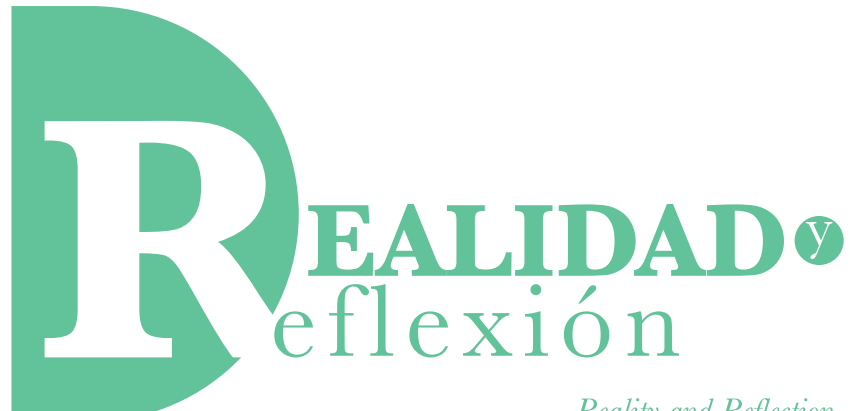

ISSN 1992-6510

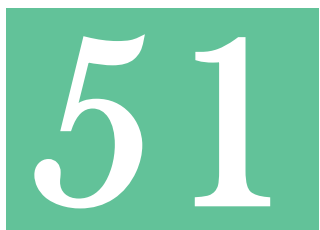

Año 20, N 51, San Salvador, El Salvador, Centroamérica. Revista Semestral Enero-Junio 2020

YEAR 20, N51, SAN SALVADOR, EL SALVADOR, CENTRAL AMERICA. SEMESTRAL JOURNAL JANUARY-JUNE 2020

\title{
Predicción de BPN a 32 Semanas de Gestación en Hospital Nacional General. Modelo De Cilindro
}

\section{LBW Prediction at 32 Weeks of Gestation in General National Hospital. Cylinder Model}

Pilar Hernández Svendblad Investigadora asociada Instituto de Ciencia, Tecnología e Innovación, Universidad Francisco Gavidia lic.pilarhd@gmail.com 


\section{RESUMEN}

El bajo peso al nacer (BPN) corresponde a la causa más importante que afecta a la mortalidad neonatal y a la morbilidad infantil. Algunos estudios muestran una fuerte relación entre el bajo peso al nacer y factores biológicos de la madre, socioeconómicos y sanitarios, por ejemplo, altura materna, pobreza y bajo número de controles prenatales, respectivamente. Debido a que estos factores no pueden ser solucionados de manera inmediata, se intentó desarrollar un modelo predictivo que los omitiera,y, además, sea accesible a los establecimientos de salud, clínicas, hospitales, independientemente si son públicos o privados, pero especialmente aquellos que no disponen de equipo tecnológico suficiente para llevar un monitoreo fetal adecuado. Para atender tal objetivo se llevó a cabo un levantamiento de datos específicos de las hojas de Historia Clínical Perinatal CLAP de los expedientes clínicos correspondientes a las madres con resultados a término con Bajo Peso al Nacer entre los años 2016 y 2018 del Hospital Nacional General de Neumología y Medicina Familiar: “Dr. José Antonio Saldaña”. Dentro de los datos tomados hubo dos variables que se consideraron ajenos a factores biológicos maternos, socioeconómicos y sanitarios: Altura uterina y Edad Gestacional. Estas dos variables han sido suficiente para construir el modelo de cilindro que nos ha ayudado a predecir, dentro de las limitaciones del estudio, el peso al nacer a 37 y 38 semanas, a partir de los datos de la edad gestacional de $32 \pm 1$ semana.

Palabras clave: Predicción del Bajo Peso al Nacer, BPN, Altura Uterina, Edad Gestacional, Modelo de cilindro, tarjeta CLAP OPS/OMS, Perímetro cefálico fetal, Bajo Peso al Nacer en E1 Salvador

\section{ABSTRACT}

Low birth weight $(L B W)$ corresponds to the most important cause that affects neonatal mortality and infant morbidity. Some studies show a strong relationship between low birth weight and maternal biological, socioeconomic, and health factors, for example, maternal height, poverty, and a low number of prenatal controls, respectively. Because these factors cannot be solved immediately, an attempt was made to develop a predictive model that would omit them, and, in addition, be accessible to health facilities, clinics, hospitals, regardless of whether they are public or private, but especially those that do not have sufficient technological equipment to carry out adequate fetal monitoring. To meet this objective, a specific data collection was carried out on the CLAP Perinatal Clinical History sheets from the clinical records corresponding to mothers with term results with Low Birth Weight between 2016 and 2018 from the National General Hospital of Pneumology and Family Medicine: "Dr. José Antonio Saldaña". Within the data collected there were two variables that were considered unrelated to maternal, socioeconomic and health biological factors: Symphysis Fundal Height and Gestational Age. These two variables have been sufficient to construct the cylinder model that has helped us to predict, within the limitations of the study, the weight at birth at 37 and 38 weeks, based on data from week $32 \pm 1$ of gestational age.

Key words: Low Birth Weight Prediction, LBW, Symphysis Fundal Height, Gestational Age, Cylinder Model, CLAP OPS/OMS sheets, Head Circumference, Low Birth Weight in El Salvador. 


\section{Introducción}

Cada año nacen más de 20 millones de niños con bajo peso al nacer (BPN) en todo el mundo. La Organización Mundial de la Salud (OMS) define el "bajo peso al nacer" como un peso al nacer inferior a $2500 \mathrm{~g}$. El objetivo de la OMS para el 2025 es reducir en un 30\% el número de niños con esta característica, pasando de 20 a 14 millones al año $[5,8]$.

La importancia del bajo peso al nacer es que corresponde a la causa más importante que afecta a la mortalidad neonatal y a la morbilidad infantil. El bajo peso al nacer puede ser consecuencia de un parto prematuro o por una restricción del crecimiento intrauterino (RCIU), o incluso una combinación de ambas condiciones. Algunos estudios muestran una fuerte relación entre el bajo peso al nacer y factores biológicos maternos, socioeconómicos y sanitarios [1,3,4,6,7,13,15, $16,20,21]$.

Existe una gran variabilidad en la incidencia del BPN según la región, incluso dentro de un mismo país. La gran mayoría de casos se dan en países de ingresos bajos y medios [6]. En Latinoamérica hay una prevalencia del 9\%, sin embargo, hay un $10 \%$ de neonatos que no son pesados al nacer [8].

De acuerdo a los datos publicados por el Ministerio de Salud desde el año 2012, se registran al menos 6500 nacidos vivos con bajo peso y al menos 600 nacidos de muy bajo peso cada año. Entre las causas que generan más egresos en la red pública de salud de neonatos (028 días) se encuentran los problemas relacionados al crecimiento fetal lento, desnutrición fetal y trastornos relacionados con la gestación corta y el bajo peso al nacer [30]. Es importante tomar en cuenta que detrás de cada nacimiento con bajo peso al nacer hay una familia en sufrimiento, por lo que lograr un manejo adecuado de este problema podría reducir este desconsuelo, así como, los recursos de estas familias y del Estado destinados al tratamiento de estos niños.

La razón de plantear este tema de estudio es esclarecer si en E1 Salvador la distribución de bajo peso puede adaptarse a un modelo matemático que permita que a partir de una gestación relativamente temprana pueda predecirse, $o$ al menos, emitir una alerta de un posible caso de bajo peso al nacer, que haga posible reconducir el crecimiento fetal de acuerdo con las guías clínicas específicas.

Un neonato con BPN puede ser debido a un parto pre término o debido a RCIU. Los RCIUs se diferencian entre, si son de crecimiento simétrico, o asimétrico. En el crecimiento simétrico existe un desfase en el crecimiento proporcional en todo el cuerpo: peso, talla y perímetro cefálico, mientras que en el crecimiento asimétrico la medida afectada es el peso. Los de crecimiento asimétrico, si se tratan a tiempo, tienen mayor probabilidad de éxito en su desarrollo $[2,6,12,18,37,38,39,40,42,44]$. Algunos estudios han evidenciado que hay mayor prevalencia de BPN con crecimiento simétrico en países desarrollados, mientras que en los países en vías de desarrollo hay mayor prevalencia de BPN con crecimiento asimétrico [6].

Los neonatos con bajo peso al nacer tienen más probabilidad de padecer complicaciones en su desarrollo. Los problemas más comunes a los 
que se enfrentan estos bebés con bajo peso son: Bajos niveles de oxígeno al nacer, incapacidad de mantener su temperatura corporal, dificultad para alimentarse y ganar peso, infecciones, problemas para respirar, problemas neurológicos como hemorragia intraventricular, trastornos intestinales como enterocolitis necrotizante, síndrome de muerte súbita del lactante, por tanto, su atención debe ser especializada hasta ganar el peso adecuado. Consecuentemente tendrían la necesidad de asistencia en unidad neonatal de cuidados intensivos, cunas con temperatura controlada, alimentación especial, tratamientos para otras complicaciones, etc. [31, 44, 45].

Es importante que los neonatos con BPN recuperen su desfase de crecimiento en los primeros cinco meses de vida ya que después no pueden lograrlo o es más costoso.

Sin embargo, las complicaciones no solo se manifiestan en el período post natal, sino también a lo largo de la infancia y adolescencia, incluso son más propensos a desarrollar condiciones crónicas. En la etapa adulta estos pacientes presentan enfermedades cardiovasculares, diabetes tipo II, osteoporosis, obesidad, muchas veces debido a la gran cantidad de proteínas que deben consumir $[36,44]$.

Se ha visto que los pacientes con BPN favorecen no solo problemas de desarrollo físico sino también alteraciones del desarrollo mental, por ejemplo, problemas de aprendizaje y lenguaje, secuelas motoras y auditivas y alteraciones de conductas $[36,44]$.

Ser un neonato con bajo peso al nacer, es una condición que acompaña durante toda la vida, es por ello que se considera importante investigar la distribución de esta condición, ya que muchos de los casos podrían ser evitados, como con la desnutrición materna [9.11], o tratados, como una infección. De esta manera, se podría evitar el sufrimiento de tantas familias y neonatos, así como, reducir los recursos destinados a estos pacientes, o a una mejor distribución de los mismos

\section{Modelo de cilindro.}

En un primer momento el estudio estaría enfocado en una curva sobre el peso materno ganado a lo largo de todo el embarazo, con el objetivo de establecer el peso mínimo que debería tener la madre al principio de cada trimestre. Dentro de los datos tomados de la hoja de historia clínica perinatal CLAP (Centro Latinoamericano de Perinatología), comúnmente denominada CLAP, se anotaron todas las medidas de los controles prenatales, donde se especifican las medidas con su edad gestacional correspondiente, incluyendo la altura uterina. Sin embargo, los datos obtenidos de las tarjetas CLAP sobre el peso antes del embarazo y al final del mismo muchas veces no es correcto. Por lo que se decidió descartar la idea.

Durante un reciente curso de Buenas Prácticas Clínicas realizado en octubre de 2019, se presentó un video sobre estudios clínicos en seres humanos donde se mostraban los fetos de diferentes semanas de gestación dentro de un bote de vidrio. A partir de este video se pensó, que, al ser estos botes de forma cilíndrica, y ver que el feto ocupaba gran parte del volumen de este, se podría obtener el volumen del feto y este podría indicarnos un peso aproximado. 
Para ello, se debía contar con dos variables, una altura y un radio. Se tomó la altura uterina como nuestra primera variable. La altura uterina mide la distancia entre el pubis y la parte superior del útero, medida en centímetros. Después de las 20 semanas, la altura del fondo uterino a menudo coincide con el número de semanas que tiene de embarazo, sin embargo, se acepta un margen de $\pm 2 \mathrm{~cm}$. Si la altura del fondo uterino tiene una medida mayor o menor de la esperada puede indicar afecciones como: RCIU, macrosomía fetal, poco líquido amniótico o demasiado líquido amniótico [27]. Para nuestra segunda variable se tomó el radio del perímetro cefálico. Sin embargo, este último no está en las hojas CLAP.

$V=\pi r^{2} h \rightarrow P n=\pi r^{2} A u$

$P n=$ Peso al nacer $(g)$

$r^{2}=$ Radio del perímetro cefálico $\left(\mathrm{cm}^{2}\right)$

$A u=$ Altura uterina 32semanas $\pm 1(\mathrm{~cm})$

Se han buscado más referencias sobre modelos de predicción del bajo peso al nacer con modelo de cilindro, y no se ha encontrado, por lo que se puede considerar un modelo nuevo.

\section{Materiales y método.}

La propuesta para este estudio fue sometida al Comité de Ética Local del hospital seleccionado y fue aprobado tanto el protocolo original como su desviación.

Así mismo, en cumplimiento de la Ley de Acceso a la Información Pública también se llevaron a cabo las instrucciones jurídicas pertinentes, a través de la Unidad Jurídica del hospital.

Se solicitó acceder a los expedientes clínicos del archivo general, correspondientes a las madres con resultados de bajo peso al nacer e igual número de expedientes correspondientes a madres con resultados de peso normal en el mismo período.

\section{Criterios de inclusión}

Grupo experimental: madres $\geq 18$ años de edad $\mathrm{y} \leq 35$ años de edad, con resultado vivos o muertos, parto uníparo por cesárea o vaginal, nacidos entre la semana de gestación 37 y 38 con un peso al nacer menor a 2500 g, nacidos entre junio 2016 y junio 2018, sin enfermedades congénitas, con al menos un control prenatal entre la semana de gestación 31 y 33 .

Grupo control: madres $\geq 18$ años de edad $y \leq 35$ años de edad, con resultados vivos o muertos, parto uníparo por cesárea o vaginal, nacidos entre la semana de gestación 37 y 38 con un peso al nacer igual o mayor a $2500 \mathrm{~g}$, nacidos entre junio 2016 y junio 2018, sin enfermedades congénitas, con al menos un control prenatal entre la semana de gestación 31 y 33.

\section{Criterios de Exclusión}

Madres menores de 15 años o mayores de 35 años, parto gemelar, hijos pre término, aquellos expedientes en los que no se contaba con controles prenatales entre la semana 31-31. También se descartaron los percentiles de altura uterina inferior al P10 y superior al P90. En un 
primer momento se establecieron otros criterios maternos como que no tuviera antecedentes de preclampsia, hipertensión, diabetes, fuese fumadora, ya sea pasiva o activa, consumiese drogas, etc. Sin embargo, no todos los CLAPs tenían este tipo de información, y, además, se consideró no discriminar entre este tipo de factores como se mencionó anteriormente.

A partir de la semana 32 el feto empieza a aumentar peso muy rápidamente, por ello, se tomó como punto de referencia la semana 32. y se dio una apertura de 1 semana más o 1 semana menos, debido a que muchas veces la semana gestacional se determina con base a la última menorrea, sin embargo, no siempre es fácilmente comprobable.

El total de casos de resultados con bajo peso al nacer entre junio 2016 a junio 2018 fueron 208. Aplicando los criterios de inclusión y exclusión a todos los casos de bajo peso al nacer para este estudio, únicamente se pudieron tomar en cuenta 19. Los casos descartados comprendían a los resultados pre término, consultas prenatales que no corresponden a nuestro margen de $32 \pm 1$ semana, datos ilegibles, partos gemelares, enfermedad congénita, expedientes que no se encontraron o que ya estaban archivados, entre otros.

\section{Herramientas}

\section{- Hoja CLAP OPS/OMS HISTORIA} CLÍNICA PERINATAL

- Tablas de medidas antropométricas fetales y maternas de OPS (Organización Panamericana de la Salud) 2011, para la vigilancia del crecimiento fetal: edad gestacional, altura uterina; edad gestacional, perímetro cefálico [12].
- Panecal Scientific Calculator versión 6.7.5. Aplicación móvil.

- Formularios de Recolección de Datos

- Excel 2016

\section{Metodología}

Para la aplicación del modelo del cilindro, en primer lugar, teníamos que obtener una segunda variable, el radio.

De acuerdo con la bibliografía consultada hay dos medidas antropométricas fetales a las que se les da seguimiento que nos podrían proporcionar el radio de nuestro cilindro: perímetro cefálico y perímetro abdominal [12]. Sin embargo, se optó por la primera, ya que en nuestro esquema del modelo del cilindro la variable que daba el grosor a nuestro feto es el perímetro cefálico. De este perímetro cefálico obtendríamos la variable $r^{2}$. ¿Pero cómo saber el perímetro cefálico de nuestra población de estudio?

Se creó un archivo de Excel 2016, en el que se creó una columna denominada EG (edad gestacional) la cual podría variar entre 31 y 33 semanas, con prioridad de escoger el dato que más se acerque a 32 semanas. Se insertó una columna llamada altura uterina, cuyo valor obtendríamos de los CLAPs. Se adicionó otra columna en la que se identificó el percentil al cual pertenecía la altura uterina, de acuerdo a la gráfica de Edad gestacional vs. Altura Uterina, del Manual de Autoinstrucción de Vigilancia Fetal OPS 2011 [12]. Basándonos en este percentil, y, asumiendo que las proporciones en las medidas de crecimiento son homogéneas, se consultó 
la gráfica de Edad Gestacional vs. Perímetro Craneal (perímetro cefálico) del mismo manual de autoinstrucción tomando como medida el dato que se encontrara en el mismo percentil análogo al de la altura uterina. Por ejemplo, si la altura uterina de $28 \mathrm{~cm}$ a 32 semanas de gestación corresponde al P50, entonces tomamos como perímetro cefálico, el dato del P50 a 32 semanas de gestación, que sería $31 \mathrm{~cm}$.

Sin embargo, la gráfica de altura uterina está divida en P10, P50 y P90, mientras que la de perímetro craneal está dividida entre P5, P50 y P95, por lo que las reglas para tomar los perímetros cefálicos se manejaron de la siguiente manera:

1. Si la altura uterina se localiza entre P10 y P50, o entre P50 y P90 equidistantemente, se toma el valor medio entre P5 y P50, y P50 y P95, respectivamente.

2. Si la altura uterina se localiza entre P10 y P50, o entre P50 y P90, pero no es un punto equidistante, se toma como valor del perímetro cefálico, el número entero más cerca del límite correspondiente. Es decir, si nuestra altura uterina es mayor que P10 y menor a $\mathrm{P} 50$, pero corresponde a un punto más cerca de $\mathrm{P} 10$, entonces nuestro perímetro cefálico es aquel número entero que le siga al P5. Si, por el contrario, la altura uterina estuviese más cerca del P50, entonces nuestro valor del perímetro cefálico sería el número entero inmediatamente por debajo del P50.

3. Si la altura uterina corresponde a P90, P50 o P10, se toma como perímetro cefálico el valor que se encuentre en P95, P50 y P5, respectivamente Tabla 1.
4. Debe redondearse el resultado del perímetro cefálico a la unidad.

\section{Tabla 1}

Au: Altura uterina; $P_{A u}:$ Percentil de altura uterina según semana gestacional; PC: Perimetro Cefálico; $P_{P i}:$ Percentil correspondiente al perímetro cefálico análogo a altura uterina; $r_{P i}:$ Radio del Perímetro cefálico con un decimal.

\begin{tabular}{|c|c|c|c|c|}
\hline $\mathrm{Au}$ & $\mathbf{P}_{\mathrm{Au}}$ & PC & Ppc & $\mathbf{r}_{\mathrm{Pc}_{\mathrm{c}}}$ \\
\hline 31 & P90 & 32 & P95 & 5.1 \\
\hline 31 & $<$ P90 & 31 & $<$ P95 & 4.9 \\
\hline 29 & P50 & 31 & $\mathrm{P} 50$ & 4.9 \\
\hline 30 & $<$ P90 & 32 & $<$ P95 & 5.1 \\
\hline 28 & $>$ P50 & 30 & $>\mathrm{P} 50$ & 4.8 \\
\hline 30 & $>\mathrm{P} 50$ & 32 & $>\mathrm{P} 50$ & 5.1 \\
\hline 28 & $<$ P50 & 31 & $>\mathrm{P} 50$ & 4.9 \\
\hline 29 & $>\mathrm{P} 50$ & 31 & $>\mathrm{P} 50$ & 4.9 \\
\hline 26 & P10 & 30 & P5 & 4.7 \\
\hline 30 & P90 & 32 & P95 & 5.1 \\
\hline 30 & $\begin{array}{c}<\mathrm{P} 90> \\
\mathrm{P} 50\end{array}$ & 32 & $\begin{array}{c}<\mathrm{P} 95> \\
\text { P50 }\end{array}$ & 5.1 \\
\hline 28 & $>$ P50 & 31 & $>\mathrm{P} 50$ & 4.9 \\
\hline 27 & $<$ P50 & 30 & $<\mathrm{P} 50$ & 4.8 \\
\hline 27 & $<\mathrm{P} 50$ & 30 & $<\mathrm{P} 50$ & 4.8 \\
\hline 28 & $\mathrm{P} 50$ & 31 & P50 & 4.9 \\
\hline 32 & $<\mathrm{P} 90$ & 34 & $<\mathrm{P} 95$ & 5.4 \\
\hline 28 & $\mathrm{P} 50$ & 31 & P50 & 4.9 \\
\hline 30 & $<\mathrm{P} 90$ & 32 & $<$ P95 & 5.1 \\
\hline 26 & $\begin{array}{c}<\mathrm{P} 50> \\
\mathrm{P} 10\end{array}$ & 29 & $\begin{array}{c}<\mathrm{P} 50> \\
\text { P5 }\end{array}$ & 4.6 \\
\hline
\end{tabular}

Habiendo obtenido los perímetros cefálicos con relación a la edad gestacional y percentil correspondiente nos disponemos a obtener el radio, asumiendo una forma circular del cráneo. 


$$
r=\frac{P C}{2 \pi}
$$

\section{$r=$ radio del perímetro cefálico $(\mathrm{cm})$ \\ $P C=$ perímetro cefálico $(\mathrm{cm})$}

Se disponen los radios correspondientes en una columna. Es importante mencionar que solo se tuvo en cuenta un decimal del radio calculado.

Debido a que la aplicación de la fórmula da como resultado volumen, y queremos obtener peso, debemos multiplicar por la densidad del feto.

De acuerdo a algunos estudios [23,41], el promedio de la densidad fetal es de $1.04 \mathrm{~g} / \mathrm{cm}^{3}$, por lo que se procedió a ajustar la fórmula. Así mismo, de acuerdo a los resultados se estableció un margen en la variación de los resultados de $\pm 250 \mathrm{~g}$, margen similar al modelo utilizado de Johnson y Toshach $( \pm 240 \mathrm{~g})$, el cual se describe más adelante. Con la conversión de volumen a peso se presentan los resultados en la Tabla 2.

\section{Resultados}

\section{$P n=\pi r^{2} A u * \partial$}

Pn=Peso al nacer $(g)$

$r=$ Radio del Perímetro cefálico $(\mathrm{cm})$

$A u=$ Altura uterina $(\mathrm{cm})$

$\partial=$ densidad fetal $=1.04\left(9 / \mathrm{cm}^{3}\right)$
Tabla 2.

Resultados de aplicación del modelo de cilindro

\begin{tabular}{c|c|c|c}
\hline $\begin{array}{c}\text { Resultado del } \\
\text { modelo (g) }\end{array}$ & $+\mathbf{2 5 0} \mathbf{g}$ & $\mathbf{- 2 5 0 g}$ & $\begin{array}{c}\text { Peso al } \\
\text { nacer (g) }\end{array}$ \\
\hline 2627.2 & 2877.2 & 2377.2 & 2400 \\
\hline 2465.5 & 2715.5 & 2215.5 & 2400 \\
\hline 2306.5 & 2556.5 & 2056.5 & 2400 \\
\hline 2542.4 & 2792.4 & 2292.4 & 2444 \\
\hline 2085.6 & 2335.6 & 1835.6 & 2200 \\
\hline 2542.4 & 2792.4 & 2292.4 & 2446 \\
\hline 2226.9 & 2476.9 & 1976.9 & 2340 \\
\hline 2306.5 & 2556.5 & 2056.5 & 2208 \\
\hline 1872.6 & 2122.6 & 1622.6 & 1750 \\
\hline 2542.4 & 2792.4 & 2292.4 & 2300 \\
\hline 2542.4 & 2792.4 & 2292.4 & 2300 \\
\hline 2226.9 & 2476.9 & 1976.9 & 2412 \\
\hline 2011.1 & 2261.1 & 1761.1 & 2200 \\
\hline 2011.1 & 2261.1 & 1761.1 & 2200 \\
\hline 2226.9 & 2476.9 & 1976.9 & 2400 \\
\hline 3061.5 & 3311.5 & 2811.5 & 2364 \\
\hline 2226.9 & 2476.9 & 1976.9 & 2440 \\
\hline 2542.4 & 2792.4 & 2292.4 & 2016 \\
\hline 1809.6 & 2059.6 & 1559.6 & 2300 \\
\hline & & & \\
\hline
\end{tabular}

De los 19 casos únicamente hubo tres que no se apegaron al modelo. Dos de estos casos tomaban datos extremos del radio de perímetro cefálico, $4.6 \mathrm{~cm}$ y $5.4 \mathrm{~cm}$. Además, de acuerdo al estudio de Kacem et al, 2013 el valor de la densidad es una media de valores tomados entre $0.95-1.18 \mathrm{~g} /$ $\mathrm{cm}^{3}$ según edad gestacional. En él se concluyó que el valor medio de densidad fetal a término es de $1.04 \mathrm{~g} / \mathrm{cm}^{3}$. Los autores manifiestan que no se vio ninguna diferencia en las densidades correspondientes a fetos sin ningún tipo de anormalidad, con los fetos desarrollándose con RCIU o bajo condiciones de diabetes gestacional, entre otras afecciones [23]. 


\section{Discusión de resultados}

Se considera que el modelo de cilindro ajustado se aproxima a los datos reales de bajo peso al nacer. Además, se da un margen de error similar al modelo propuesto por Johnson y Toshach, utilizado para calcular el peso fetal del embarazo antes del nacimiento en pacientes con embarazo a término. En 1954 Johnson y Toshach propusieron un método para medir la altura uterina y se le aplicó una fórmula de constantes del estudio de 200 casos, donde relaciona la altura uterina con la presentación fetal, con un resultado de variación del peso fetal $\pm 240 \mathrm{~g}$ en $68 \%$ de los recién nacidos $[17,43]$.

Es importante mencionar que múltiples estudios relacionan el bajo peso al nacer a factores biológicos maternos, sanitarios $\mathrm{y}$ socioeconómicos, por lo que esta influencia no cuantificable es la que nos podría dar la variación. Además, al ser la altura uterina y el perímetro cefálico dos variables que son medidas por ojo humano son sujetas a error [10,14,27,29,30,33].

Con respecto a la muestra estudiada de pesos normales, se evidencia que los parámetros de altura uterina y perímetro cefálico, al menos, hasta las 33-34 semanas de gestación no nos arrojan una diferencia respecto a los de bajo peso, pero sí nos pueden indicar el peso mínimo que se proyecta del resultado en el nacimiento. Adicionalmente a este cálculo se debe hacer un seguimiento en las próximas semanas que nos dé una señal de alerta con el fin de clasificar el feto con restricción de crecimiento, aplicar el modelo y reconducirlo a un nivel normal, ya sea por una infección no tratada, poco líquido amniótico, desnutrición materna, o tomar otras decisiones obstétricas [45]. Por ejemplo, de acuerdo al Manual de OPS para la Vigilancia fetal [12], después de la semana 32 el perímetro abdominal del feto, tanto para RCIU simétrico como asimétrico, cae por debajo del percentil 5, siendo el indicador más sensible.

Esto con el fin de reducir el número de recién nacidos con bajo peso, ya que, se considera que si se tiene un diagnóstico con suficiente tiempo para tratarse muchos de estos casos pueden evitarse.

\section{Aplicación}

Como factores que favorecen al bajo peso al nacer tenemos: Edad materna, estado nutricional de la madre, salud de la madre, tabaquismo, consumo de bebidas alcohólicas, anemia materna, hipertensión arterial, antecedentes de otros nacimientos con BPN, acortamiento del período entre embarazos, incompetencia del cuello uterino, infección cérvico-vaginal, factores socioeconómicos, factores de estrés, raza, por mencionar algunos. Desafortunadamente, no hay ningún tratamiento para el $\mathrm{BPN}$, solo la identificación temprana y manejo de estos factores y la vigilancia fetal podrían evitar el nacimiento con bajo peso al nacer [22].

En este sentido, es de utilidad, cuando no se dispone de un aparato de ultrasonografía, que generalmente se da en lugares donde la asistencia sanitaria es de escasos recursos, identificar, en primer lugar, si la paciente tiene alguno o varios de estos factores de riesgo mencionados anteriormente. Ya sea mediante una entrevista, exámenes analíticos, examen físico, etc. Así mismo, valorar la altura uterina de acuerdo a las tablas de crecimiento fetal. Posteriormente, si 
nuestra paciente tiene entre 31-33 semanas de gestación podemos aplicar el modelo de cilindro y calcular el peso con el que el bebé nacerá a 37 semanas de gestación, en el que ya se considera a término. $\mathrm{Al}$ ser un amplio rango de peso, $500 \mathrm{~g}$, tomamos el límite inferior del rango y decidimos si el mínimo peso es suficiente para que el bebé se desarrolle sin mayor complicación. Si el límite inferior no es aceptable para un bebé a término de 37 semanas, esto nos alertará sobre los posibles riesgos que estén influenciando en su peso, por ejemplo: Desnutrición materna, se podría hacer un plan de nutrición específico para el embarazo; hipertensión, se administrarían medicamentos para la enfermedad de la madre, sin embargo, estos también podrían afectar al feto; situación de estrés, podría tomarse en cuenta una incapacidad mayor, menor horario laboral o probablemente identificar un problema de violencia; anemia materna, se le administrarían los suplementos necesarios, los cuales se deben tomar antes, durante y después del embarazo; infección cervico-vaginal, se ha descrito que el $22 \%$ de BPN se deben a infección, se iniciaría el tratamiento. El manejo de estos factores a 5-6 semanas antes del parto aumenta la probabilidad de reconducir el crecimiento fetal, ya que, en casos en los que se identifica un retraso del crecimiento y sufrimiento fetal a 35 o 36 semanas probablemente la única solución es administrar medicamentos para madurar los pulmones e interrumpir el embarazo.

Si, por el contrario, el peso mínimo que puede obtener el feto a la semana 37 roza los $2500 \mathrm{~g}$ y no se aprecia un sufrimiento fetal la opción podría ser continuar con el embarazo bajo una vigilancia fetal muy de cerca.
Así mismo, una vez confirmado el bajo peso al nacer, ya sea por RCIU o por pre término, $y$, dependiendo del valor que nos arroje el modelo, también se tomarán en valoración aquellas necesidades de las que se debe disponer para ofrecer al feto la mejor atención. Por ejemplo, iniciar el trabajo de parto en un hospital que esté adecuado para este tipo de situaciones: que cuenten con cunas térmicas, método de madre canguro, fuente de oxígeno, monitor de frecuencia, equipo de intubación, entre otros [31].

Como se mencionó anteriormente, este modelo es una parte de la evaluación y vigilancia fetal integral. Entre más información se tenga, se podrá mejorar la estrategia de manejo del bajo peso al nacer.

\section{Limitaciones}

En primer lugar, se tiene que mencionar que estos datos se obtuvieron del CLAP de un hospital de la red nacional. Los médicos y enfermeras que ayudan a llenar este tipo de formularios atienden a muchos pacientes por hora lo que pone en riesgo al profesional sanitario de estar en completa capacidad para hacer esta tarea de manera efectiva. También se debe tomar en cuenta, que el CLAP es llenado, de acuerdo al apartado que les corresponda, por otros centros asistenciales, por lo que un error en la unidad de salud, se continua hacia el momento del parto y luego postparto. Motivo por lo que esta tarjeta CLAP está sujeta a muchos errores: error con la edad gestacional debido a que la paciente no recuerda su última menstruación, hay error en la medición de la altura uterina, las fechas no coinciden con el número de semanas, 
los campos no están completamente llenos, etc. Consecuentemente hay cierta incertidumbre de estos datos oficiales.

En segundo lugar, en el momento que se fue a confirmar diagnósticos de RCIU con el tamaño acorde del feto y el peso comparado con la edad gestacional, se encontraron algunos casos que hicieron dudar de la precisión de los mismos. Por ejemplo, en los que el recién nacido tuvo un peso de menos de 2000 gramos, con 38 semanas de gestación, y se tenía marcado como el que tamaño fetal era acorde y no tenía RCIU, otros casos marcaban que el tamaño fetal era acorde, pero también marcaba que tenía RCIU, o, al contrario, por lo que se decidió no tomar en cuenta los diagnósticos confirmatorios. Motivo por el que no se ha podido caracterizar la distribución de RCIU.

Tercero, el estudio también está limitado a las tablas de OPS 2011 [12], cuya población muestral fue obtenida en países de América del Sur, por lo que la población de El Salvador probablemente no encaje del todo en los percentiles que se escogen. Sin embargo, se hizo este ejercicio con las tablas recientes de OMS 2017 [24], NICHD [26], e INTERGROWTH-21st Project [34,35] pero fueron las tablas de OPS las que mejor resultado dieron con el modelo.

Otra de las limitaciones del estudio ha sido el bajo número de casos con los que se ha podido correr este modelo, ya que no está establecido de manera obligatoria las consultas prenatales a las $32 \pm 1$ semanas de gestación, o simplemente no hay un seguimiento de los embarazos de acuerdo a lo recomendado. Se encontraron CLAPs que únicamente tenía un control prenatal, y muchos no tenían ni uno.

\section{Recomendaciones}

1. Promover las consultas prenatales en los establecimientos de salud.

2. Oficializar un esquema de controles prenatales de acuerdo a las semanas clave en las que se puedan identificar algún problema en el crecimiento, por ejemplo, semana 24-25 (cuando cae el perímetro cefálico en RCIU simétrico), semana 32-33 (cuando cae el perímetro abdominal en ambas RCIUs), etc.

3. Capacitación para llenar un CLAP y del valor nacional de estos datos.

4. Control de calidad de los datos detallados.

5. Desarrollar tablas de crecimiento fetal para el país

6. Control de calidad por parte del Ministerio de Salud para analizar los datos obtenidos de todos los establecimientos de salud que alimentan las estadísticas nacionales, ya que, sobre estos datos se hacen las políticas de salud.

\section{Conclusiones}

El modelo de cilindro propuesto por primera vez en este estudio para calcular el peso al nacer, $y$ predecir el bajo peso del recién nacido a término desde la semana de gestación $32 \pm 1$, se considera que puede ser útil como una señal de alerta. A pesar que, dentro de este período, en cuanto a altura uterina y perímetro cefálico, no hay diferencia con los resultados de peso normal, puede ser una herramienta más que complete el panorama gestacional del feto. Una vez determinado que el 
feto tiene alguna restricción en su crecimiento u otra anomalía, este modelo puede servir para proyectar su peso a término y tomar las medidas oportunas para reconducir un crecimiento adecuado con suficiente tiempo de antelación.

A raíz de estos resultados sería conveniente realizar este modelaje a nivel nacional, de carácter prospectivo, con los siguientes parámetros establecidos: determinación de edad gestacional por ecografía, con primer control prenatal antes de las diez semanas, medición del perímetro cefálico y altura uterina a las 32 semanas de gestación y compararlo con el resultado al nacer. Así como, hacerlo de manera retrospectiva, deseable con datos realizados por ecografía. Esto tendría doble función, desarrollar tablas que caractericen el crecimiento fetal en El Salvador, tanto de peso normal como de bajo peso, así como, comprobar o ajustar el modelo propuesto [2,19, 27].

\section{Agradecimientos}

Especial agradecimiento a Dra. Claudia Cecilia Hernández Barillas y Dr. Ricardo Ernesto Hurtado Navas por su asesoramiento sobre el tema, Personal del Hospital Nacional General de Neumología y Medicina Familiar: "Dr. José Antonio Saldaña”, Licenciado Luis Alejandro Rivera Flores por asesoramiento sobre la interpretación de datos, Ing. Carlos Alberto Hernández Deleón y Dr. Marlio Paredes Gutiérrez por la revisión del documento.

\section{Referencias}

1. Avila-Rosas, H., Casanueva, E., \& Barrera, A. (1988). Algunos determinantes biológicos y sociales del peso al nacer. Salud pública de México, 30(1), 47-53.
2. Beca, J. P., Rizzardini, M., Weldt, E., Fernandez, M. D. P., Bloomfield, J., \& Corvalán, S. (1989). Talla, peso y perímetro craneano según edad gestacional en recién nacidos de menos de 35 semanas. Revista chilena de pediatría, 60(1), 1-5.

3. Bello Luján, L. M., Saavedra Santana, P., Gutiérrez García, L. E., García Hernández,J. Á., \& Serra Majem, L. (2015). Características sociodemográficas y sanitarias asociadas con bajo peso al nacer en Canarias. Nutrición Hospitalaria, 32(4), 1541-1547.

4. Bernis, C. (2005). Determinantes biológicos y culturales del peso al nacer en España 2000: valoración en hijos de mujeres inmigrantes y no inmigrantes. Antropo, 10(1), 61-73.

5. Blencowe, H., Cousens, S., Chou, D., Oestergaard, M., Say, L., Moller, A. B., ... \& Lawn, J. (2013). Born too soon: the global epidemiology of 15 million preterm births. Reproductive health, 10(1), S2.

6. Bortman, M. (1998). Factores de riesgo de bajo peso al nacer. Revista panamericana de salud pública, 3, 314-321.

7. -Castrillón, J. J., Giraldo-Cardona, J. F., Murillo-Díaz, C. A., Jordán-Ordóñez, Y., Orozco-Arango, J. A., Robledo-Vásquez, P., ... \& Villegas-Arenas, Ó. A. (2008). Relación entre peso al nacer y algunas variables biológicas y socioeconómicas de la madre en partos atendidos en un primer nivel de complejidad en la ciudad de Manizales, Colombia, 1999 al 2005. Revista Colombiana de Obstetricia y Ginecología, 59(1), 20-25. 
8. de la Salud, A. M. (2011). Hacia la reducción de la mortalidad perinatal y neonatal (No.WHA64. 13). Organización Mundial de la Salud

9. de la Salud, O. M. (2017). Metas mundiales de nutrición 2025: documento normativo sobre retraso del crecimiento (No. WHO/NMH/ NHD/14.3). Organización Mundial de la Salud.

10. Engstrom, J. L., Sittler, C. P., \& Swift, K. E. (1994). Fundal height measurement: Part 5The effect of clinician bias on fundal height measurements. Journal of nurse-midwifery, 39(3), 130-141.

11.FAO, OPS, WFP y UNICEF. 2018. Panorama de la seguridad alimentaria y nutricional en América Latina y el Caribe 2018. Santiago. Número de páginas (132) . Licencia: CC BY-NC-SA 3.0 IGO.

12. Fescina, R. H., Schwarcz, R. I. C. A. R. D. O., \& Diaz, A. G. (2011). Vigilancia del crecimiento fetal: manual de autoinstrucción. (2a Ed.) Centro Latinoamericano de Perinatología y Desarrollo Humano.

13. Franco Monsreal, J., Cobos, T., del Ruby, M., Hernández Gómez,J. R., \& Serralta Peraza,L. E.D.S. (2018). Factores de riesgo de bajo peso al nacer según el modelo de regresión logística múltiple. Estudio de cohorte retrospectiva en el municipio José María Morelos, Quintana Roo, México. Medwave, 18(01).

14. Freire, D. M. C., Cecatti, J. G., \& Paiva, C. S. M. (2010). Symphysis-fundal height curve in the diagnosis of fetal growth deviations. Revista de Saúde Pública, 44, 1031-1038.
15. Gamboa Delgado, E. M., Rangel Díaz, Y.A., \& Gutiérrez Gómez, Y.Y.(2017). Asociación entre peso al nacer y factores de riesgo cardiometabólicos en niños de Bucaramanga, Colombia. Nutrición Hospitalaria, 34(5), 1105-1111.

16. García Andrés, R., Valerio Rodríguez, F. T., Casuscelli, L., Barón Mizzi, I., \& García Moreno, M. (2016). Variabilidad del peso de los recién nacidos según el país de origen materno. Pediatría Atención Primaria, 18(72), 303-310.

17. García, C. S., Piña, F. G., \& Juárez, G. G. (2007). Utilidad del método de Johnson y Toshach para calcular el peso fetal en embarazos de término en un hospital de segundo nivel. Ginecol Obstet Mex, 75, 317-24.

18. González, R. (2010). Salud materno-infantil en las américas. Revista chilena de obstetricia y ginecología, 75(6), 411-421.

19. Gutiérrez Pineda, M. B. (2015). Perfil del neonato que nace en el Hospital Nacional Saldaña en el período de enero a diciembre de 2013 (Doctoral dissertation, Universidad de E1 Salvador).

20. Hachuel, L. S., Boggio, G. S., \& Wojdyla, D. (2004). Modelos logit mixtos: una aplicación en el área de la salud.

21. Ibarra, K. H., Cuadro, D. R., Manotas, E. N., Castro, C. C., \& Vanegas, S. N. (2015). Análisis de los factores de riesgo de bajo peso al nacer a partir de un modelo logístico polinómico. Prospectiva, 13(1), 76-85. 
22. Infogen (2016). Bajo Peso al Nacer. Recuperado de: https://infogen.org.mx/ bajo-peso-al-nacer/. Última vez consultado. 14 de diciembre 2019.

23. Kacem, Y., Cannie, M. M., Kadji, C., Dobrescu, O., Lo Zito, L., Ziane, S., ... \& Staelens, R. (2013). Fetal weight estimation: comparison of two-dimensional US and MR imaging assessments. Radiology, 267(3), 902-910.

24. Kiserud, T., Piaggio, G., Carroli, G., Widmer, M., Carvalho, J., Jensen, L. N., .. \& Benachi, A. (2017). The World Health Organization fetal growth charts: a multinational longitudinal study of ultrasound biometric measurements and estimated fetal weight. PLoS medicine, 14(1), e1002220.

25. Las matemáticas en el feto (2011). Recuperado de: http://epsomat.blogspot. com/2011/10/1as-matematicas-en-elfeto.html. Última vez consultado $22 \mathrm{de}$ noviembre 2019

26. Louis, G. M. B., Grewal, J., Albert, P. S., Sciscione, A., Wing, D. A., Grobman, W. A., ... \& Nageotte, M. P. (2015). Racial/ethnic standards for fetal growth: the NICHD Fetal Growth Studies. American journal of obstetrics and gynecology, 213(4), 449-e1.

27. Martinelli, S., Bittar, R. E., \& Zugaib, M. (2001). Proposta de nova curva de altura uterina para gestações entre a $20 \mathrm{a}$ e a $42 \mathrm{a}$ Semana. $R B G O, 23(4)$.
28. Mayo Clinic (2017). Recuperado de: https:// www.mayoclinic.org/es-es/healthy-lifestyle/ pregnancy-week-by-week/expert-answers/ fundal-height/faq-20057962. Última vez consultado el 24 de noviembre 2019.

29. Meler, E., Peralta, S., Figueras, F., Eixarch, E., Coll, O., Puerto, B., \& Cararach, V. (2005). Altura uterina: curvas de normalidad y valor diagnóstico para un bajo peso neonatal. Progresos de Obstetricia y Ginecología, 48(10), 480-486.

30. Ministerio de Salud (2018).Recuperado de: https://www.transparencia.gob.sv/institutions/ minsal. Última vez consultado 14 de junio 2019.

31. Ministerio de Salud. Dirección General de Promoción y Prevención. Guía de Atención del Bajo Peso al Nacer. Recuperado de https://www.minsalud.gov.co/sites/ $\mathrm{rid} /$ Lists/BibliotecaDigital/RIDE/VS/ PP/13Atencion\%20del\%20Bajo\%20peso.pdf. Visitado última Vez 14 de diciembre 2019.

32. Morse, K., Williams, A., \& Gardosi, J. (2009). Fetal growth screening by fundal height measurement. Best Practice \& Research Clinical Obstetrics E' Gynaecology, 23(6), 809818.

33. Neilson, J. P. (2007). Medición de la altura uterina durante el embarazo. Base de Datos Cochrane de Revisiones Sistemáticas, (4).

34. Papageorghiou, A. T., Ohuma, E. O., Altman, D. G., Todros, T., Ismail, L. C., Lambert, A., ... \& Noble, J. A. (2014). 
International standards for fetal growth based on serial ultrasound measurements: the Fetal Growth Longitudinal Study of the INTERGROWTH-21st Project. The Lancet, 384(9946), 869-879.

35. Papageorghiou, A. T., Ohuma, E. O., Gravett, M. G., Hirst, J., Da Silveira, M. F., Lambert, A., ... \& Bertino, E. (2016). International standards for symphysisfundal height based on serial measurements from the Fetal Growth Longitudinal Study of the INTERGROWTH-21st Project: prospective cohort study in eight countries. bmj, 355, i5662.

36. Pérez-Escamilla, R., \& Pollitt, E. (1992). Causas y consecuencias del retraso del crecimiento intrauterino en América Latina.

37. Pidre, M. C., Azpeitia, G. G., \& Ortega, F. D. (2001). Análisis de la nutrición y del crecimiento en los recién nacidos de muy bajo peso [Microforma]. Universidad de Las Palmas de Gran Canaria.

38. Puig Palau, A. (2017). Evaluación del crecimiento posnatal en los prematuros de muy bajo peso con edad gestacional menor o igual a 32 semanas desde el nacimiento hasta los 5 años de vida.

39. Rodríguez, O. T., Malaverry, H., Páucar, M., Romero, E., Broncano, J., Aquino, R., \& Gamarra, R. (2011). Curva de crecimiento intrauterino y su aplicación en el diagnóstico de restricción del crecimiento intrauterino. Revista Peruana de Ginecología y Obstetricia, 57(2), 69-76.
40. Sepúlveda, S. E., Crispi, B. F., Pons, G. A., \& Gratacos, S. E. (2014). Restricción de crecimiento intrauterino. Revista Médica Clinica Las Condes, 25(6), 958-963.

41. Toro-Ramos, T., Paley, C., Pi-Sunyer, F. X., \& Gallagher, D. (2015). Body composition during fetal development and infancy through the age of 5 years. European journal of clinical nutrition, 69(12), 1279-1289.

42. Van der Velde, J., Caputo, A., \& Illia, R. (1994). Retardo del crecimiento intrauterino Guía para diagnóstico y tratamiento. Rev. Hosp. Mat. Inf. Ramón Sardá, 13(2), 60-65.

43. Vega Forero, D. I., \& Medina Moncayo, M. L. (2014). Coeficiente de concordancia del peso fetal estimado por el método de Johnson y Toshach y el peso de neonatos nacidos en un hospital público de Bogotá (Doctoral dissertation, Universidad Nacional de Colombia).

44. Velázquez Quintana, N. I., Zárraga, M. Y., Luis, J., \& Ávila Reyes, R. (2004). Recién nacidos con bajo peso; causas, problemas y perspectivas a futuro. Boletín Médico del Hospital Infantil de México, 61(1), 73-86.

45. World Health Organization. (2016). WHO recommendations on antenatal care for a positive pregnancy experience. World Health Organization.

46. Yepes, H. A. C. (2008). Restricción del crecimiento intrauterino. Memorias Curso de Actualización en Ginecología y Obstetricia, 16, 213. 